\title{
Changes in Ankle Brachial Pulse Wave Velocity during a Five-Year Follow-up Period in Older Japanese Adults: Sub-analysis Results of the Health Research Volunteer Study in Japan
}

\author{
Nobutaka Doba ${ }^{1}$, Yasuharu Tokuda ${ }^{2}$, Hirofumi Tomiyama ${ }^{3}$, Nathan E. Goldstein ${ }^{4}$, \\ Toshio Kushiro $^{5}$ and Shigeaki Hinohara ${ }^{6}$
}

\begin{abstract}
Objective Brachial-ankle pulse wave velocity (baPWV) is a measure of arterial stiffness. However, precisely how aging, hypertension and other factors influence this in progressively stiffening large arteries, especially in older adults, remains uncertain. We examined changes in arterial stiffness in a population of active older Japanese adults using a five-year follow-up cohort study.

Methods Comprehensive geriatric assessments were performed in socially active adults over the age of 70 years at The Life Planning Center Clinic in Tokyo. Clinically acceptable baPWV measurements at baseline and at the end of the study were obtained in 257 individuals (115 men, baseline mean age of $78 \pm 4$ years; 142 women, baseline mean age of $77 \pm 4$ years) classified into four groups based on the use of antihypertensive interventions at the end of the study: Group 1 (112 normotensives), Group 2 (49 hypertensives without medication use), Group 3 (39 hypertensives with medication use initiated during the follow-up period) and Group 4 (57 hypertensives receiving medications throughout the follow-up period).

Results During the follow-up period, Group 1 exhibited no changes in blood pressure with increases in baPWV. Group 2 exhibited increases in blood pressure and baPWV. Both Groups 3 and 4 exhibited decreases in blood pressure without any changes in baPWV. Other factors such as age, gender and the hemoglobin level could also have influenced baPWV.

Conclusion Arterial stiffness increases with aging; however, antihypertensive medications were found to provide protective effects against the development of arterial stiffness during a five-year follow-up period. Other factors that modify baPWV were also identified.
\end{abstract}

Key words: aging, arterial stiffness, antihypertensive medication, older adults, low hemoglobin

(Intern Med 52: 21-27, 2013)

(DOI: 10.2169/internalmedicine.52.8671)

\section{Introduction}

In Japan, the percentage of individuals over the age of 65 years had reached $22.5 \%$ as of 2010 (1). In 1898, Sir William Osler stated that longevity depends on vascular issues and that "a man is as old as his arteries and many deaths arise primarily or secondarily as a result of vascular issues" (2). Aging of the vascular system is thought to progress as a result of either predetermined genetic factors or changeable environmental factors caused mostly by detrimental lifestyle choices. Aging is associated with decreases in arterial compliance that can result in systolic hypertension (3).

\footnotetext{
${ }^{1}$ Division of Research and Education, Life Planning Center Foundation, Japan, ${ }^{2}$ Institute of Clinical Medicine, Graduate School of Comprehensive Human Sciences, University of Tsukuba, Japan, ${ }^{3}$ The Second Department of Internal Medicine, Tokyo Medical University, Japan, ${ }^{4}$ Brookdale Department of Geriatrics and Palliative Medicine, Mount Sinai School of Medicine and the James J Peters VA Medical Center, USA, ${ }^{5}$ Center for Health Evaluation and Promotion, Nihon University School of Medicine, Japan and ${ }^{6}$ Life Planning Center Foundation, Japan Received for publication July 25, 2012; Accepted for publication August 27, 2012 Correspondence to Dr. Nobutaka Doba, doba@krd.biglobe.ne.jp
} 
Many studies have reported that arterial stiffness itself is an independent risk for cardiovascular events, separate from other conventional factors (4-6). Pulse wave velocity measurements are the gold standard for assessing arterial stiffness (7-9). Hypertension has a strong effect on the progression of arterial stiffness and its associated adverse outcomes. This relationship explains why hypertension is an important predictor of cardiovascular events and why controlling high blood pressure results in significant reductions in cardiovascular events and mortality (10-12). However, the effects of aging, hypertension, pharmaceutical intervention and other various factors on arterial stiffness remain to be explored, especially among older adults $(13,14)$. We therefore chose to examine the effects of medication and other factors on arterial stiffness during a five-year cohort study primarily examining the prediction of frailty syndrome development in older Japanese adults. In this study, pulse pressure was determined to be a predictor associated with three other factors: slow walking speed and cognitive and hearing deficits $(15,16)$.

\section{Materials and Methods}

\section{Study population sample}

In 2000, the "New Elderly Citizen Movement" was initiated at the Life Planning Center Foundation in Tokyo, Japan, with the goal of encouraging older adults to change their lifestyles so as to become more active and productive. The details of the subjects' health conditions and healthrelated life behaviors have been reported elsewhere $(17,18)$. The study began in 2002, and 407 members without frailty based on a standardized definition $(19,20)$ were voluntarily enrolled by the end of 2005. In this manuscript, we report the results of a secondary analysis of the "Health Research Volunteer Study (HRVS)" to examine the effects of aging, hypertension, the use of antihypertensive medications and other factors on arterial stiffness. Among the initial 407 subjects enrolled in the HRVS (184 men with a mean age of $78 \pm 4$ years, 223 women with a mean age of $77 \pm 4$ years), 34 dropped out, 22 died, 65 developed clinical frailty syndrome and 29 developed clinical conditions for which pulse wave measurements were inappropriate for assessment (e.g. atrial fibrillation, frequent ectopic beats, ankle brachial index less than 0.95). These 150 subjects were therefore excluded from the subanalysis. Most of the participants had their own family doctors and received their medical care outside of the HRVS. Subjects with systolic blood pressure greater than $140 \mathrm{mmHg}$ were considered to be hypertensive in this study.

At the end of the follow-up period, 257 subjects were classified into four groups based on the systolic blood pressure level and the use of pharmaceutical interventions: Group 1 with normal systolic blood pressure $(\leq 139 \mathrm{mmHg})$ $(n=112)$, Group 2 with high systolic blood pressure $(\geq 140$ $\mathrm{mmHg}$ ) without medication use $(\mathrm{n}=49)$, Group 3 with medication use initiated during the follow-up period $(n=39)$ and
Group 4 with medication use throughout the entire study period $(n=57)$.

Written informed consent was obtained from all participants, and ethical permission was obtained from the Ethics Committee of the Japan Society of Health Evaluation and Promotion.

\section{Assessments}

All of the subjects underwent a comprehensive geriatric assessment (CGA) at both baseline and study termination (five years after enrollment). The details of the CGA have been reported elsewhere (16). All of the data elements, including physical and psychosocial variables, were obtained from a self-reported structured demographic and healthrelated questionnaire, which included a comprehensive inventory of medications carefully reviewed with the patients by trained nurses.

Data were obtained at the time of each subject's visit to the Life Planning Center Clinic in Tokyo and included the following elements: height $(\mathrm{cm})$, weight $(\mathrm{kg})$, body mass in$\operatorname{dex}(\mathrm{BMI})$, maximum mid-arm circumference $(\mathrm{mm})$, blood pressure (systolic, diastolic, pulse pressures; $\mathrm{mmHg}$ ), heart rate (bpm), brachial ankle pulse wave velocity (baPWV: $\mathrm{cm} /$ sec) and ankle brachial index (ABI) (PWV/ABI obtained using the BP-203RPEII-Colin device). All blood pressure readings were obtained as part of the baPWV measurement from the right side of the upper arm. The baPWV measurements were obtained while the subjects were in a supine position after having rested for 10 minutes. Laboratory tests included a complete blood cell count (CBC) and the levels of hemoglobin $(\mathrm{g} / \mathrm{dL})$, creatinine $(\mathrm{mg} / \mathrm{dL})$, estimated glomerular filtration rate $(\mathrm{eGFR})\left(\mathrm{mL} / \mathrm{min} / 1.73 \mathrm{~m}^{2}\right)$, total cholesterol $(\mathrm{mg} /$ $\mathrm{dL})$, hemoglobin A1c $(\%)$, total serum protein $(\mathrm{g} / \mathrm{dL})$ and $\mathrm{c}-$ reactive protein $(\mathrm{CRP}, \mathrm{mg} / \mathrm{dL})$. The level of B-type natriuretic peptide (BNP, ng/mL) was examined only at the end of the study period.

\section{Analysis}

The data were analyzed using SPSS version 15.0J (Tokyo, Japan). Parametric data were expressed as the mean $\pm 1 \mathrm{SD}$ and analyzed using paired $t$-tests for comparisons of the baseline and follow-up test results. The nonparametric parameters were analyzed using the chi-square test. Comparisons among four parameters were performed using a oneway analysis of variance to obtain the F-value, and the Bonferroni and Sidak methods were applied to confirm the significance of differences between each pair of two values at baseline and at the end of the study among the four groups. A linear stepwise regression analysis was performed to explore significant variables associated with baPWV at the end of the study period. A two-tailed p-value less than 0.05 was regarded as significant.

\section{Results}

The study population is described in Table 1 . The mean 
Table 1. Demographic Characteristics of the Population and Subgroups

\begin{tabular}{lcccccc}
\hline & $\begin{array}{c}\text { Group 1 } \\
(n=112)\end{array}$ & $\begin{array}{c}\text { Group 2 } \\
(n=49)\end{array}$ & $\begin{array}{c}\text { Group 3 } \\
(n=39)\end{array}$ & $\begin{array}{c}\text { Group 4 } \\
(n=57)\end{array}$ & $\begin{array}{r}\text { F-value } \\
\text { or } \mathrm{X}^{2}\end{array}$ & p-value \\
\hline Age at Baseline & $77 \pm 4$ & $78 \pm 6$ & $77 \pm 4$ & $77 \pm 4$ & 0.532 & 0.660 \\
Age at Study Termination & $82 \pm 4$ & $83 \pm 4$ & $82 \pm 4$ & $83 \pm 4$ & 0.547 & 0.650 \\
Sex (f/m) & $60 / 52$ & $26 / 23$ & $19 / 20$ & $37 / 20$ & 3.048 & 0.384 \\
Follow-up duration (months) & $63 \pm 5$ & $63 \pm 3$ & $63 \pm 3$ & $63 \pm 3$ & 0.474 & 0.701 \\
Hypercholesterolemia-2 (a/p) & $100 / 12$ & $44 / 5$ & $34 / 5$ & $48 / 9$ & 1.119 & 0.772 \\
Diabetes Mellitus-2 (a/p) & $101 / 11$ & $47 / 2$ & $36 / 3$ & $53 / 4$ & 2.980 & 0.821 \\
Smoking-2 (nsm/exsm/sm) & $100 / 8 / 4$ & $47 / 1 / 1$ & $38 / 1 / 0$ & $52 / 3 / 2$ & 4.198 & 0.650 \\
\hline
\end{tabular}

Group 1 were subjects who were normotensive at end and never received medications. Group 2 were subjects who were hypertensive at beginning or became so during course of study but never received medications. Group 3 were those who were hypertensive at some point in the study and who were started on antihypertensive medications at some point during the study. Group 4 were subjects who were hypertensive and on medications at baseline and who received medications throughout the entire course of the study

Abbreviations: f: female, m: male, a: absent, sp: p, present, nsm: non-smoker, exsm: ex-smoker, sm: smoker

Table 2. Morphometric Characteristics of Subgroup Populations

\begin{tabular}{lcccccc}
\hline & Group 1 $(\mathrm{n}=112)$ & Group 2 $(\mathrm{n}=49)$ & Group 3 $(\mathrm{n}=39)$ & Group 4 $(\mathrm{n}=57)$ & F-value & p-value \\
\hline Height-1 & $157 \pm 8$ & $157 \pm 8$ & $156 \pm 8$ & $153 \pm 8$ & 3.771 & 0.011 \\
Height-2 & $155 \pm 8 * *$ & $156 \pm 8 * *$ & $154 \pm 9 * *$ & $151 \pm 9 * *$ & 3.879 & 0.010 \\
\hline Weight-1 & $54 \pm 9$ & $54 \pm 9$ & $55 \pm 11$ & $56 \pm 9$ & 0.562 & 0.641 \\
Weight-2 & $52 \pm 9 * *$ & $52 \pm 10 * *$ & $54 \pm 11 * *$ & $53 \pm 9 * *$ & 0.452 & 0.716 \\
\hline BMI-1 & $21.9 \pm 2.6$ & $22.9 \pm 2.5$ & $22.7 \pm 2.9$ & $23.9 \pm 3.4$ & 7.110 & 0.001 \\
BMI-2 & $21.6 \pm 2.9 * *$ & $21.2 \pm 2.7 * *$ & $22.3 \pm 3.1 * *$ & $23.3 \pm 3.2 * *$ & 5.833 & 0.001 \\
\hline UAC-1 & $25.9 \pm 2.4$ & $25.9 \pm 2.4$ & $26.3 \pm 2.3$ & $27.1 \pm 2.8$ & 3.021 & 0.030 \\
UAC-2 & $25.0 \pm 2.5 * *$ & $24.7 \pm 2.8 * *$ & $25.5 \pm 2.7 * *$ & $25.9 \pm 3.4 * *$ & 1.913 & 0.128 \\
\hline
\end{tabular}

1) Abbreviations and related units

BMI: Body Mass Index, UAC: Upper Arm Circumference (cm)

2) 1: baseline, 2: end of study (after 5 years)

3) $*<0.05$ 、* $*<0.01$ (paired t-test: 1 vs. 2)

age at the time of study entry was $77 \pm 4$ years, and the mean duration of follow-up was $63 \pm 5$ months. There were no differences at the end of the study among the four groups with regard to age, sex, follow-up duration (months), treatment for diabetes mellitus and hypercholesterolemia or smoking status. Differences were noted throughout the study in terms of significantly lower heights and larger body mass indices (BMIs) in Group 4 than in Groups 1 and 2. No differences in weight were observed among the four groups throughout the study period. Only the upper arm circumference was larger at baseline in Group 4 than in Group 1 (Table 2).

At baseline (Table 3, Figs. 1, 2), comparisons of the parameters, including systolic blood pressure, diastolic blood pressure, pulse pressure and baPWV, among the four groups revealed an increasing trend from Groups 1 to 4, with significant differences revealed using a one-way analysis of variance. The ABI also decreased slightly, but significantly from Groups 1 to 4 . In contrast, no significant differences were observed in the heart rate among the four groups.

There were significant differences in the blood pressure and vascular parameters at the end of the five-year followup period (Table 3, Figs. 1, 2). In Group 1, the systolic, diastolic and pulse pressures and heart rates exhibited no sig- nificant changes in association with significant increases in baPWV and decreases in ABI. In Group 2, the systolic blood pressures, diastolic blood pressures, pulse pressures and baPWV revealed statistically significant increases in association with decreases in ABI without changes in heart rate. In both Groups 3 and 4, the systolic and diastolic blood pressures decreased significantly without any changes in either the pulse pressure or baPWV. Only ABI showed slight but significant decreases in Group 3.

In terms of comparisons between the groups at baseline using the Bonferroni and Sidak methods, significant differences in systolic pressure, diastolic pressure and pulse pressure were observed between Group 1 and the other three groups; however, no other significant differences were observed among the other between-group comparisons (Fig. 1). A significant difference in ABI was observed between Group 1 and Group 4 only. Regarding baPWV, significant differences were observed between Group 1 and the other three groups; however, no other significant differences were observed among the other between-group comparisons (Fig. 2).

At the end of the study (Table 3, Fig. 1), significant differences in the systolic pressure were observed between 
Table 3. Blood Pressure and Related Characteristics

\begin{tabular}{|c|c|c|c|c|c|c|}
\hline & Group 1(n=112) & Group 2(n=49) & Group 3(n=39) & Group $4(n=57)$ & F-value & p-value \\
\hline SBP-1 & $126 \pm 13$ & $137 \pm 17$ & $140 \pm 16$ & $144 \pm 20$ & 17.717 & 0.001 \\
\hline SBP-2 & $124 \pm 13$ & $153 \pm 15^{* *}$ & $130 \pm 16^{* *}$ & $135 \pm 17 * *$ & 44.696 & 0.001 \\
\hline DBP-1 & $74 \pm 9$ & $79 \pm 9$ & $80 \pm 11$ & $82 \pm 13$ & 8.528 & 0.001 \\
\hline DBP-2 & $73 \pm 12$ & $84 \pm 10 * *$ & $74 \pm 12 * *$ & $76 \pm 10 * *$ & 13.470 & 0.001 \\
\hline PP-1 & $52 \pm 8$ & $58 \pm 12$ & $60 \pm 11$ & $62 \pm 13$ & 11.917 & 0.001 \\
\hline PP-2 & $52 \pm 9$ & $69 \pm 11 * *$ & $56 \pm 10$ & $59 \pm 12$ & 29.446 & 0.001 \\
\hline baPWV-1 & $1704 \pm 268$ & $1963 \pm 382$ & $1906 \pm 356$ & $1928 \pm 375$ & 10.221 & 0.001 \\
\hline baPWV-2 & $1819 \pm 320^{* *}$ & $2248 \pm 378^{* *}$ & $2022 \pm 572$ & $1949 \pm 347$ & 29.446 & 0.001 \\
\hline ABI-1 & $1.17 \pm 0.07$ & $1.16 \pm 0.08$ & $1.15 \pm 0.100$ & $1.13 \pm 0.09$ & 3.114 & 0.027 \\
\hline ABI-2 & $1.15 \pm 0.09 * *$ & $1.13 \pm 0.07 * *$ & $1.11 \pm 0.14 *$ & $1.11 \pm 0.08$ & 2.631 & 0.063 \\
\hline HR-1 & $69 \pm 10$ & $70 \pm 10$ & $70 \pm 10$ & $70 \pm 12$ & 0.149 & 0.930 \\
\hline HR-2 & $71 \pm 10$ & $71 \pm 11$ & $70 \pm 12$ & $69 \pm 12$ & 0.540 & 0.655 \\
\hline \multicolumn{7}{|c|}{$\begin{array}{l}\text { 1) Abbreviations and units } \\
\text { SBP: systolic blood pressure (mmHg), DBP: diastolic blood pressure }(\mathrm{mmHg}), \mathrm{PP}: \text { pulse } \\
\text { pressure, (mmHg), baPWV: brachial ankle pulse wave velocity (cm/sec), ABI: ankle } \\
\text { brachial index), HR: heart rate }(\mathrm{bpm})\end{array}$} \\
\hline
\end{tabular}

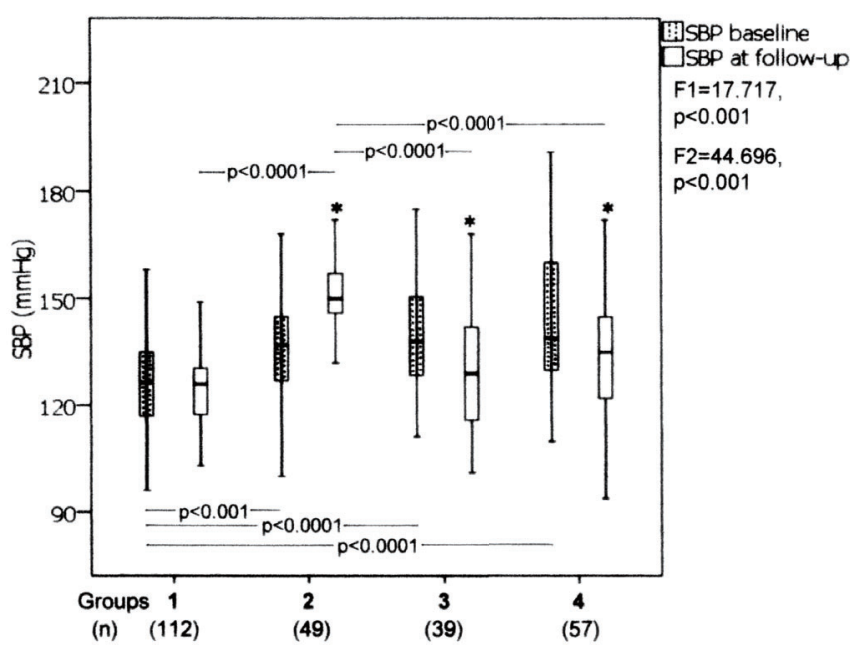

Figure 1. Comparisons of systolic blood pressure at baseline and follow-up in each pair of groups and then among the four groups (box and whisker plot). The *signifies a significant difference $(p<0.05)$ between each group at time of study entry and at study termination. The $\mathrm{F}$-values are for the comparison among the four groups at study entry (F1) and at the end of study (F2).

Group 2 and the other three groups, and also between Groups 1 and 4. No significant differences were observed between Groups 1 and 3 or between Groups 3 and 4. Significant differences in diastolic pressure were also observed between Group 2 and each of the other three groups. No significant differences were observed between pairs of any of the other three groups. The pulse pressures in Group 2 were significantly different from those in any of the other groups, and a significant difference was also observed between Groups 1 and 4. No significant differences in ABI were observed among any pairs of groups. The baPWV values in Group 2 were significantly different from those in the other three groups (Table 3, Fig. 2). No significant differ- ences were observed between any pairs of the other three groups.

During the five-year follow-up period, there were no significant differences in the other serum parameters among the four groups at baseline, with only a few exceptions (Table 4). First, the serum total protein levels tended to increase from Group 1 to Group 4, both at baseline and at the end of the study period. Comparisons between pairs of the four groups revealed a significant difference between Groups 1 and 4 only. The other exception was the eGFR, for which significant differences were observed among the four groups when using a one-way analysis of variance at the end of the study period; comparisons of each pair of two groups revealed a significant difference between Groups 1 and 4 only.

A linear stepwise regression analysis was performed to explore the significant variables associated with PWV at the end of the study period. The following parameters were examined for the analyses: age, gender, both initial and final blood pressure (BP) parameters along with other possible confounding parameters including diabetes mellitus, hypercholesterolemia, smoking status and serum markers measured at the end of the study period, as listed in Table 4. The following five factors were determined to be significant: age, gender, systolic blood pressure, the hemoglobin level measured at the end of the study period and diastolic blood pressure measured at study entry (Table 5).

Concerning medication use, calcium channel blockers (CCBs, 71\%) and angiotensin receptor blockers (ARBs, $51 \%$ ) were prescribed in the majority of treated patients, and diuretics were prescribed in $11 \%$ of the cases. Other drugs, including angiotensin-converting enzyme (ACE) inhibitors, $\alpha \beta$ blockers, $\alpha_{1}$ blockers and $\beta$ blockers, were used in less than $8 \%$ of the cases. Combined use of CCBs and ARBs, the most common prescription pattern in Japan, was seen in $27 \%$ of the cases. The mean number of medications was $1.6 \pm 0.7$. No significant differences were observed between 


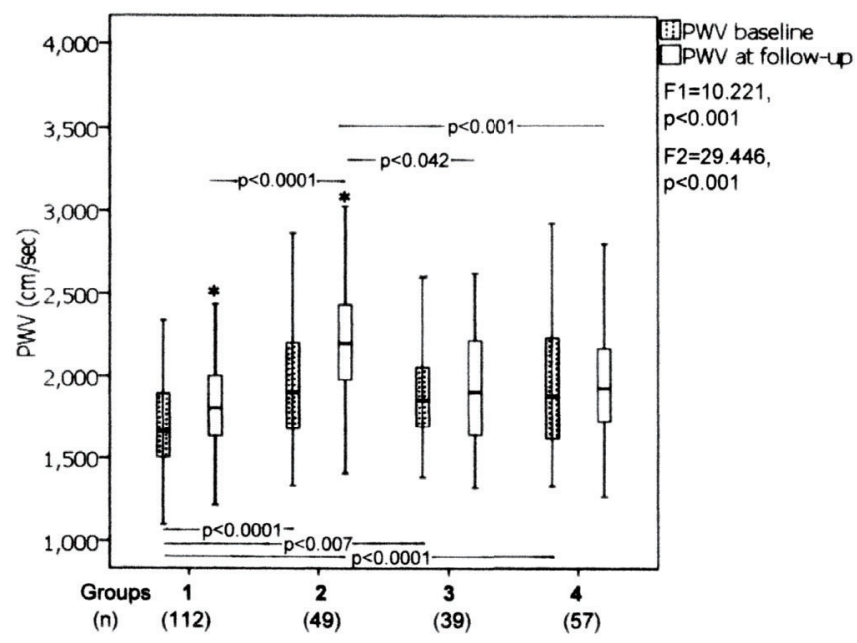

Figure 2. Comparisons of baPWV at baseline and follow-up in each pair of the four groups and then among the four groups (box and whisker plot). The *signifies a significant difference $(p<0.05)$ between each group at time of study entry and then at study termination. The F-values are for the comparison among the four groups at baseline (F1) and at followup (F2).

Groups 3 and 4 at the end of the study period with regard to the type of antihypertensive medication prescribed.

\section{Discussion}

In aging societies, there is a tremendous focus on treating hypertension among older adults. Until recently, however, little reliable data have supported the effects and benefits of lowering blood pressure in this population. However, the Hypertension in the Very Elderly Trial (HYVET) recently provided strong evidence for the benefits of antihypertensive medication use among relatively healthy patients over the age of 80 years, demonstrating a significant reduction in cardiovascular morbidity and overall mortality $(12,21)$. Additionally, economic benefits of antihypertensive treatment for patients over the age of 80 years have also been reported (22). Aging and high blood pressure are widely accepted as independent risk predictors of arterial stiffness and cardiovascular morbidity and mortality (9-11). However, how these two and other parameters influence progressively stiffening larger arteries, especially in older adults, remains to be explored.

We found that the subjects in Group 1 (normotensives, by definition) exhibited no changes in systolic, diastolic or pulse pressures; however, they exhibited significant increases in baPWV without demonstrating any changes in heart rate. This result implies that the increases in baPWV observed in this group arose from increases in arterial stiffness related to aging of the arterial walls. Comparisons between Groups 2 and 3 provided important data regarding the clinical management of hypertension. Another relevant finding is that there were no significant differences in baPWV among Groups 1, 3 and 4 at the end of the study period. This result suggests that antihypertensive medication use may prevent increases in baPWV arising from high blood pressure, thus helping to maintain baPWV at the age-appropriate levels. However, the interactive mechanisms underlying the effects of blood pressure, aging and medication use on arterial stiffness could not be precisely determined using this study design.

After the five-year follow-up period, no significant differences in any other measured nonvascular parameters were observed among the four groups, with some exceptions, including eGFR. A one-way analysis of variance revealed significant differences in eGFR-2 among the four groups, with Group 4 exhibiting the lowest value. This finding suggests that the eGFR in subjects with long-lasting hypertension cannot be reversed with antihypertensive medications alone. A linear stepwise regression analysis examining the variables significantly associated with PWV at the time of study termination revealed several factors associated with baPWV. The association with hemoglobin was unexpected and further study is needed to characterize this result $(23,24)$.

There are a few limitations that must be considered when interpreting our results. First, the participants in our study were self-selected from a group of older adults with a particular interest in maintaining their health (as evidenced by their membership in the Association of New Elderly Citizen Movement in Japan) (18), which may have resulted in a possible selection bias. Second, determining medication compliance and obtaining information regarding changes in medication use during the course of the study was difficult. Since each participant had his or her own physician prescribe the antihypertensive medications, the treatments may have been modified throughout the course of the study. Therefore, the effects of treatment could not be specified in terms of each class of medication. The exact length of antihypertensive treatment could not be precisely determined, and the only definitive conclusion that can be made is that the length of treatment was less than five years in Group 3 and more than five years in Group 4. Finally, the details of the medications used at the end of the study period are related to current trends in prescribing among primary care physicians in Japan. Since almost all of the generic types of antihypertensive medications have been shown to be equally effective and safe, it may thus be possible that the most important issue is lowering blood pressure within or below the normal range as opposed to simply treating patients with hypertension $(25,26)$.

\section{Conclusion}

This study helps shed new light on the ways that aging, hypertension and other selected factors shown in this study influence the progression of stiffening of large arteries in older adults. This work in older Japanese adults demonstrates that over a five-year period, arterial stiffness increases and antihypertensive medications may exert protective effects on the stiffness that occurs as a result of hyper- 
Table 4. Serum Markers of Subgroups

\begin{tabular}{|c|c|c|c|c|c|c|}
\hline & Group $1(n=112)$ & Group 2(n=49) & Group 3(n=39) & Group 4(n=57) & F-value & $\mathrm{p}$-value \\
\hline $\mathrm{Hgb}-1$ & $13.6 \pm 1.2$ & $13.4 \pm 1.3$ & $13.9 \pm 1.4$ & $13.4 \pm 1.3$ & 1.514 & 0.211 \\
\hline $\mathrm{Hgb}-2$ & $12.9 \pm 1.6^{* *}$ & $12.9 \pm 1.3 * *$ & $13.1 \pm 1.6^{* *}$ & $12.7 \pm 1.2 * *$ & 0.671 & 0.571 \\
\hline tChol-1 & $213 \pm 37$ & $222 \pm 32$ & $223 \pm 34$ & $216 \pm 30$ & 1.447 & 0.230 \\
\hline tChol-2 & $209 \pm 35$ & $215 \pm 34$ & $216 \pm 32$ & $211 \pm 34$ & 0.659 & 0.578 \\
\hline Creatinine-1 & $0.78 \pm 0.20$ & $0.80 \pm 0.24$ & $0.87 \pm 0.25$ & $0.81 \pm 0.26$ & 1.520 & 0.210 \\
\hline Creatinine-2 & $0.76 \pm 0.23$ & $0.72 \pm 0.16^{* *}$ & $0.80 \pm 0.21^{*}$ & $0.84 \pm 0.26$ & 3.050 & 0.029 \\
\hline eGFR-1 & $66 \pm 16$ & $66 \pm 18$ & $61 \pm 17$ & $62 \pm 16$ & 1.365 & 0.254 \\
\hline eGFR-2 & $66 \pm 16$ & $68 \pm 12$ & $63 \pm 15$ & $58 \pm 17 *$ & 4.493 & 0.004 \\
\hline tProt-1 & $7.1 \pm 0.4$ & $7.2 \pm 0.4$ & $7.2 \pm 0.2$ & $7.3 \pm 0.4$ & 4.222 & 0.006 \\
\hline tProt-2 & $7.2 \pm 0.4^{*}$ & $7.2 \pm 0.4$ & $7.2 \pm 0.4$ & $7.4 \pm 0.4$ & 2.919 & 0.034 \\
\hline HbAlc-1 & $5.2 \pm 0.7$ & $5.2 \pm 0.4$ & $5.4 \pm 0.9$ & $5.3 \pm 0.6$ & 1.105 & 0.348 \\
\hline $\mathrm{HbA} 1 \mathrm{c}-2$ & $5.4 \pm 0.6 * *$ & $5.4 \pm 0.5^{* *}$ & $5.5 \pm 0.7$ & $5.5 \pm 0.6 * *$ & 0.911 & 0.446 \\
\hline CRP-1 & $0.08 \pm 0.10$ & $0.09 \pm 0.11$ & $0.11 \pm 0.16$ & $0.10 \pm 0.20$ & 0.538 & 0.657 \\
\hline CRP-2 & $0.11 \pm 0.23$ & $0.29 \pm 1.12$ & $0.12 \pm 0.14$ & $0.14 \pm 0.20$ & 1.369 & 0.253 \\
\hline BNP-2 & $52 \pm 55$ & $64 \pm 49$ & $51 \pm 36$ & $68 \pm 65$ & 1.548 & 0.203 \\
\hline \multicolumn{7}{|c|}{$\begin{array}{l}\text { 1) Abbreviations and units: } \mathrm{Hgb} \text { : hemoglobin }(\mathrm{g} / \mathrm{dL}) \text {, tChol: total cholesterol }(\mathrm{mg} / \mathrm{dL}), \text { eGFR: } \\
\text { estimated glomerular filtration rate }\left(\mathrm{mL} / \mathrm{min} 1.73 \mathrm{~m}^{2}\right) \text {, tProt: total serum protein }(\mathrm{g} / \mathrm{dL}), \mathrm{HbA} 1 \mathrm{c} \text { : } \\
\text { hemoglobin } \mathrm{A} 1 \mathrm{c}(\%) \text {, CRP: c-reactive protein }(\mathrm{mg} / \mathrm{dL}) \text {, BNP: brain natriuretic peptide }(\mathrm{ng} / \mathrm{mL}) \\
\text { 2) } 1 \text { : baseline, } 2 \text { : at time of study termination } \\
\left.\text { 3) }{ }^{*} \mathrm{p}<0.05,{ }^{* *} \mathrm{p}<0.001 \text { (paired t-test: } 1 \text { vs. } 2\right) \text {. }\end{array}$} \\
\hline
\end{tabular}

Table 5. Results of Linear Stepwise Regression Analysis Exploring Relationship of Significant Independent Variables with Pulse Wave Velocity at Time of Study Termination

\begin{tabular}{lccccc}
\hline $\begin{array}{c}\text { Significant } \\
\text { variables }\end{array}$ & \multicolumn{2}{c}{ Beta coefficient } & \multicolumn{2}{c}{$95 \%$ CI of Beta } & p-value \\
\hline & Beta & SE & Lower limit & Upper limit \\
SBP at follow-up & 8.679 & 1.237 & 6.241 & 11.116 & $<0.001$ \\
Age & 33.672 & 5.724 & 22.397 & 44.947 & $<0.001$ \\
DBP at baseline & 6.593 & 2.187 & 2.284 & 10.901 & 0.003 \\
Hg at follow-up & 37.442 & 15.301 & 7.304 & 67.581 & 0.015 \\
Male gender & -93.253 & 45.269 & -182.417 & -4.089 & 0.04 \\
\hline
\end{tabular}

Abbreviations: pulse wave velocity (PWV, cm/sec), SBP (systolic blood pressure, $\mathrm{mmHg}$ ), diastolic blood pressure (DBP, $\mathrm{mmHg}$ ); hemoglobin $(\mathrm{Hg}, \mathrm{g} / \mathrm{dL})$, standard error $(\mathrm{SE})$, confidence interval $(\mathrm{Cl})$.

tension. Currently available antihypertensive agents are capable of preventing arterial stiffening, even among hypertensive octogenarians, and this has important implications for treating even the oldest adults. Further study is needed among subgroups, especially those similar to Group 2, to determine the optimal timing of initiating antihypertensive interventions as well as which medications may be the most effective.

The authors state that they have no Conflict of Interest (COI).

\section{Acknowledgement}

This work was supported by an institutional research fund from the Life Planning Center (a non-profit organization). We thank all the Healthy Research Volunteers who participated in this study, and we also express our gratitude to the other research members at the LPC for their professional and technical assistance with the study.

\section{References}

1. A profile of older Japanese 2010. International Longevity Center, Japan.
2. Lim MA, Townsend RR. Arterial compliance in the elderly: Its effect on blood pressure measurement and cardiovascular outcomes. Clin Geriatr Med 25: 192-205, 2009.

3. Stokes GS. Management of hypertension in the elderly patient. Clin Interv Aging 4: 379-389, 2009.

4. Nakano H, Okazaki K, Ajiro Y, Suzuki T, Oba K. Clinical usefulness of measuring pulse wave velocity in predicting cerebrovascular disease: evaluation from a cross-sectional and longitudinal follow-up study. J Nihon Med Sch 68: 490-497, 2001.

5. Morita Y, Nakamura Y, Miura K, Ueshima H. Brachial-ankle pulse wave velocity predicts all-cause mortality in the general population: findings from the Takashima study, Japan. Hypertens Res 33: 922-925, 2010.

6. Hatanaka R, Obara T, Watabe D, et al. Association of arterial stiffness with silent cerebrovascular lesions: the Ohasama study. Cerebrovasc Dis 31: 329-337, 2011.

7. Blacher J, Safer M. Large-artery stiffness, hypertension and cardiovascular risk in older patients. Nature Clin Pract Cardiovasc Med 2: 450-455, 2005.

8. Laurent S, Cockcroft J, Van Bortel L, et al. European network for non-invasive investigation of large arteries. Expert consensus document on arterial stiffness: methodological issues and clinical applications. Eur Heart J 27: 2588-2605, 2006.

9. Najjar SS, Scuteri A, Shetty V, et al. Pulse wave velocity is an independent predictor of the longitudinal increase in systolic blood pressure and of incident hypertension in the Baltimore Longitudi- 
nal Study of Aging. J Am Coll Cardiol 51: 1377-1383, 2008.

10. Vlachopoulos C, Aznaouridis K, Stefanadis C. Prediction of cardiovascular events and all-cause mortality with arterial stiffness: a systematic review and meta-analysis. J Am Coll Cardiol 30; 55: 1318-1327, 2010

11. Benetos A, Buatois S, Salvi P, et al. Blood pressure and pulse wave velocity values in the institutionalized elderly aged 80 and over: baseline of the PARTAGE study. J Hypertens 28: 41-50, 2010.

12. Zeglin MZ, Pacos J, Bisognano JD. Hypertension in the very elderly: Brief review of management. Cardiol J 16: 379-385, 2009.

13. Bulpitt CJ, Beckett NS, Peters R, et al. Baseline characteristics of participants in the Hypertension in the Very Elderly Trial (HYVET). Blood Press 18: 17-22, 2009.

14. O'Rourke MF, Nabasivatam M. Treatment of hypertension in patients 80 years of age or older. Minerva Med 100: 25-38, 2009.

15. Lang P-O, Michel J-P, Zekry D. Frailty syndrome: A transitional state in a dynamic process. Gerontology 55: 539-549, 2009.

16. Doba N, Tokuda Y, Goldstein NE, Kushiro T, Hinohara S. A pilot trial to predict frailty syndrome: the Japanese health Reserach Volunteer Study. Exper Getontol 47: 638-643, 2012.

17. Hinohara $S$, Doba $N$. The future profile of health promotion and disease prevention in Japan. Methods Inf Med 44: 342-347, 2005.

18. Doba $\mathrm{N}$, Hinohara $\mathrm{S}$, Yanai $\mathrm{H}$, et al. The New Elder Citizen Movement in Japan. In: Faces of Aging: The Lived Experiences of the Elderly in Japan. Matsumoto Y, Ed. Stanford University Press, Stanford, 2011: 36-59.

19. Fried LP, Tangen CM, Walston J, et al; Cardiovascular Health
Study Collaborative Research Group. Frailty in older adults: evidence for a phenotype. J Gerontol A Biol Sci Med Sci 56: M146M156, 2001.

20. Rockwood K, Song X, MacKnight C, et al. A global clinical measure of fitness and frailty in elderly people. CMAJ 173: 489495, 2005.

21. Mancia G, Grassi G. Management of very elderly hypertensives: the HYVET study. Aging Clin Exper Res 20: 494-495, 2009.

22. Szucs TD, Waeber B, Tomonaga Y. Cost-effectiveness of antihypertensive treatment in patients 80 years or older in Switzerland: an analysis of the HYVET study from a Swiss perspective. J Hum Hypertens 24: 117-123, 2010.

23. Kawamoto R, Tabara Y, Kohara K, et al. A slightly low hemoglobin level is beneficially associated with arterial stiffness in Japanese community-dwelling women. Clin Exp Hypertens 34: 92-98, 2012.

24. Jia EZ, An FH, Liu P, et al. Relationship between brachial-ankle pulse wave velocity and cardiovascular risk factors: a multi-ethnic study. Intern Med 51: 537-543, 2012.

25. Shannon R, Chaudhry M. Effect of alpha1-adrenergic receptors in cardiac pathophysiology. Am Heart J 152: 842-852, 2006.

26. Czernichow S, Zanchetti A, Turnbull F, et al; Blood Pressure Lowering Treatment Trialists' Collaboration. The effects of blood pressure reduction and of different blood pressure-lowering regimens on major cardiovascular events according to baseline blood pressure: meta-analysis of randomized trials. J Hypertens 29: 4-16, 2011.

(C) 2013 The Japanese Society of Internal Medicine http://www.naika.or.jp/imonline/index.html 